\title{
Report on Modifications to the BX12 and BX13 BC1 Dipoles ${ }^{1}$
}

\author{
J. Welch, S. DeBarger, P. Emma, A. Fisher, N. Li, J. Wu
}

December 12, 2007

\footnotetext{
${ }^{1}$ Work supported in part by the DOE Contract DE-AC02-76SF00515. This work was performed in support of the LCLS project at SLAC.
} 


\section{Contents}

1 Introduction 1

2 Modifications 1

2.1 Observations . . . . . . . . . . . . . . . . . 1

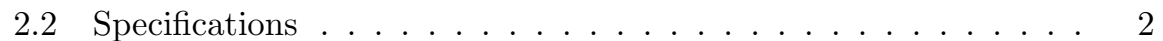

2.3 Modeling . . . . . . . . . . . . . . . . . . . 3

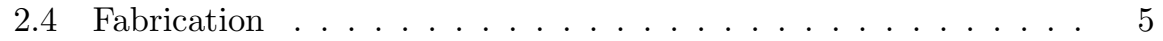

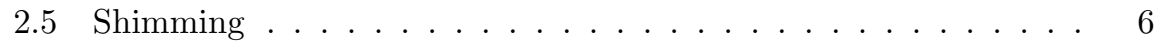

3 Measurements and Tests $\quad 7$

3.1 Field Quality . . . . . . . . . . . . . . . . . 7

3.2 Field Strength . . . . . . . . . . . . . . . . . . 8

3.3 Vacuum Chamber Effect . . . . . . . . . . . . . . . . . 11

3.4 Assembly Test . . . . . . . . . . . . . . . . . . . . . 15

3.5 Thermal Tests . . . . . . . . . . . . . . . . 15

4 Beam Calculations $\quad 16$

\section{Introduction}

Emittance growth seen during the last commissioning run in the bunch compressor optics section, $\mathrm{BC} 1$, was blamed on inadequate dipole field quality. The significant linear and nonlinear field non-uniformities generated large horizontal dispersion errors beyond $\mathrm{BC} 1$. The linear dispersion after $\mathrm{BC} 1$ was corrected using two small 'corrector' quadrupoles placed in $\mathrm{BC} 1$ for this purpose, but the remaining nonlinear field caused growth of the normalized horizontal emittance of $40 \%$ or more. At best $\gamma \epsilon_{x}$ went from $1.2 \mu \mathrm{m}$ before BC1 up to $1.7 \mu \mathrm{m}$ after $\mathrm{BC} 1$. The problem was magnified by the larger-than-design energy spread in BC1 due to a long initial bunch length.

To improve the field quality we decided to modify the two 'inner dipoles', BX12 and BX13, of the four magnet chicane during the four month down time in the Fall of 2007. Only the two inner dipoles were chosen because of the limited time available and the fact that the beam is particularly sensitive to field quality of the inner dipoles due to its very large transverse size when going through them. The modifications were completed in November and included new poles and a new pinning scheme. The outer dipoles were left unchanged.

\section{Modifications}

\subsection{Observations}

During the 2007 commissioning run measurements made with the accelerator beam led us to suspect that there might be a problem with the $\mathrm{BC} 1$ dipoles, 
so during a ROD day in June the installed (unmodified) BC1 dipoles were inspected. In BX12 we found that the top and bottom halves of the yoke were not making good contact; there was a gap between them large enough that a piece of paper could be slid through it. When the bolts holding the top and bottom halves together were tightened (they were not initially tight) the gap reduced and the paper no longer would go through. During the Fall 2007 downtime the installed dipoles were checked again and no new gaps were found.

To install the magnets it had been necessary to disassemble them into two halves, after the final magnetic measurements had been made, and then reassemble them in the tunnel. If there was a difference in the way the halves went back together, then one might expect the magnetic field obtained in the tunnel might have been different from that measured in the laboratory. The fabrication drawings showed that the locations for the holes for the pins were dimensioned independently for the two halves, as if yoke halves of different dipoles were interchangeable. The tolerance on the distance between the holes was much greater than the fit tolerance on the pins in the holes, so there was no reason to expect to be able to pin the two halves together without some kind of distortion. In fact, when BX12 and BX13 were disassembled and removed for modifications, we found obvious distortions of one of the pin holes on each of the magnets; the plane of contact bulged out and the hole was visibly out of round. In addition the four screws that held the yoke halves together were found to be all ground to custom length, and they appeared to be a bit too long. The thread engagement went right up to the last one or two threads, so these screws might not have been pulling the halves together before they ran out of thread. We also found quite a bit of steel filings in the threaded holes, which we took as evidence of galling or thread damage. Finally, we observed some pulling up of the threads from the mating surfaces. These observations confirmed our suspicions that the pinning scheme was problematic, and we implemented a new pinning scheme as part of the modifications.

The problems with joining the two halves of the magnet could explain the beam measurements that showed a large linear distortion of the field. However, the effect of the linear field error was corrected for by adjusting a pair of corrector quadrupoles in the chicane, so this was not the cause of the emittance growth. That had to be from the non-linear distortion of the field.

\subsection{Specifications}

Field quality specifications for the $\mathrm{BC} 1$ magnets were originally given in terms of maximum allowed multipole coefficients. ${ }^{1}$ A comparison of specifications and

${ }^{1}$ Multipole coefficients $b_{n}$ are defined by:

$$
\int_{-\infty}^{\infty} B_{y}(x, y=0, z) d z=\sum_{n=0}^{\infty} b_{n}\left(\frac{x}{R_{r e f}}\right)^{n}
$$

where $B_{y}$ is the main deflecting component of the magnetic field and for simplicity it is assumed the field has top/bottom symmetry with respect to the bend plane. $R_{r e f}$ is a normalizing reference radius chosen for convenience. 
Table 1: Specification in terms of the multipole coefficients with a reference radius of $20 \mathrm{~mm}$ given in the Physics Requirements Document (PRD), the Engineering Specifications Document (ESD) used to order the magnets, and measured for BX12 (typical) at SLAC .

\begin{tabular}{cccc}
\hline \hline Coefficient & PRD & ESD & SLAC Measured \\
\hline$\left|b_{1} / b_{0}\right|$ & 0.002 & 0.0002 & 0.002 \\
$\left|b_{2} / b_{0}\right|$ & 0.0005 & 0.0005 & 0.008 \\
$\left|b_{3} / b_{0}\right|$ & 0.001 & - & 0.003 \\
$\left|b_{4} / b_{0}\right|$ & 0.002 & 0.002 & 0.006 \\
\hline
\end{tabular}

SLAC measurements of the unmodified BX12 dipole is given in Table 1, and clearly shows that the SLAC-measured BX12 magnet did not meet the original specifications. The measurements of the other dipoles were similar. Measurements were also made by the manufacturer before shipping. These were done with a $15 \mathrm{~mm}$ radius rotating coil instead of a translating wire, and indicated the magnets met did meet specifications. The cause for the inconsistency between the measurements by manufacturer and SLAC is not understood. In any case, a new target specification for the modifications was adopted. Rather than specify the field quality in terms of limits on multipole coefficients, the new specification simply required that the integrated field is constant within $\pm 5 \times 10^{-4}$ over $\pm 26 \mathrm{~mm}$. The new specification was selected, in part, to account for the fact that the beam was about $30 \%$ longer than expected and consequently had more energy spread and a larger transverse size when it went through the inner dipoles.

\subsection{Modeling}

We undertook a program of modeling to understand the field quality performance of the unmodified dipoles and to design modifications for better field quality. The gap-to-length ratio of the dipoles was $43.5 / 150=0.29$, so $2 \mathrm{D}$ calculations of the field profile were not very accurate and the modeling effort was done mostly using 3D models. Two different programs were used to evaluate the models, one at SLAC based on ANSYS, and one by collaborators at ORNL using TOSCA. The $3 \mathrm{D}$ models were able to predict the measured integrated field strength distribution of the unmodified dipoles to within a few parts per thousand. Accuracy was limited by mesh size and computer memory. A comparison of the model predictions and the measured field distribution for the unmodified BX14 is given in Figure 1.

It was felt that the good-field region in the original design was not large enough and that widening the pole was desirable. The main task of the modeling effort was then to determine the best overall width of the pole. The models results are shown in Figure 2. As the pole was made wider the field quality 


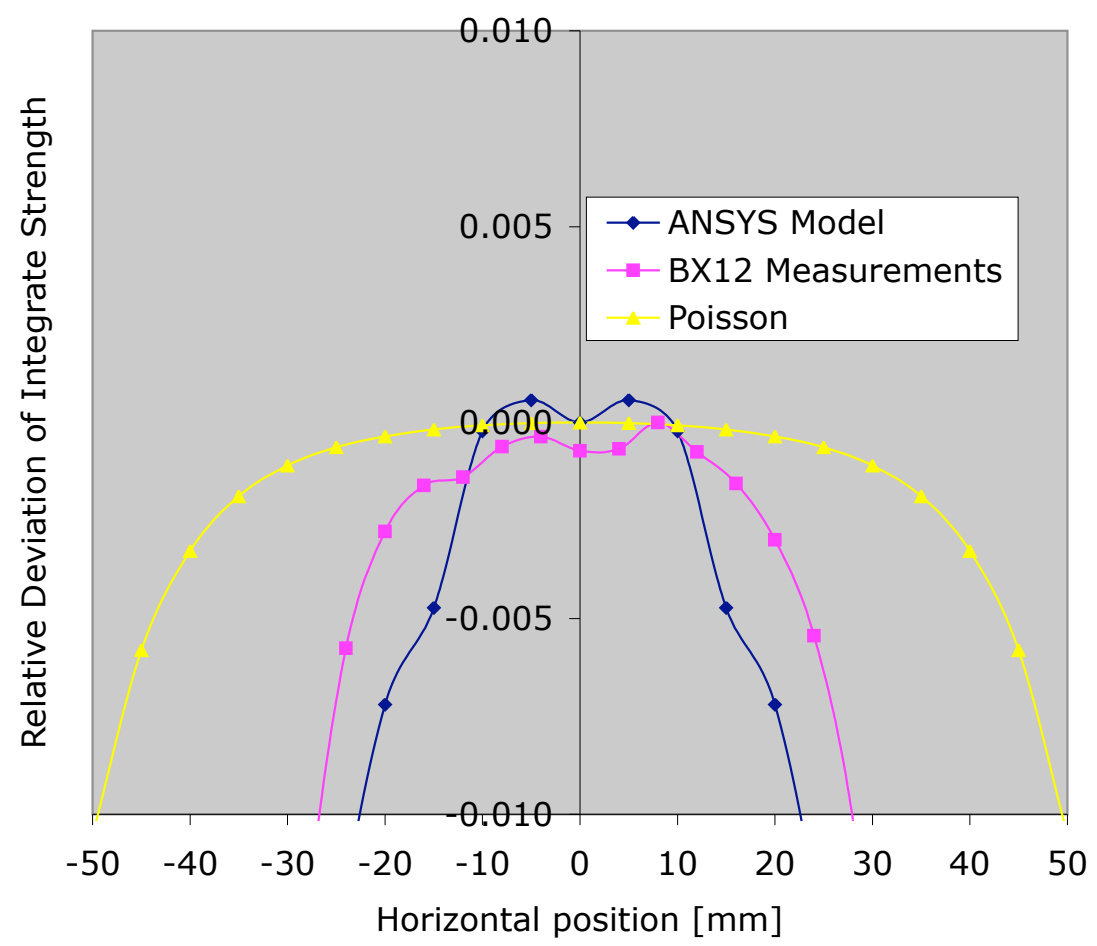

Figure 1: Comparison of 2D (Poisson) and 3D (ANSYS) calculations with the measured field uniformity. 


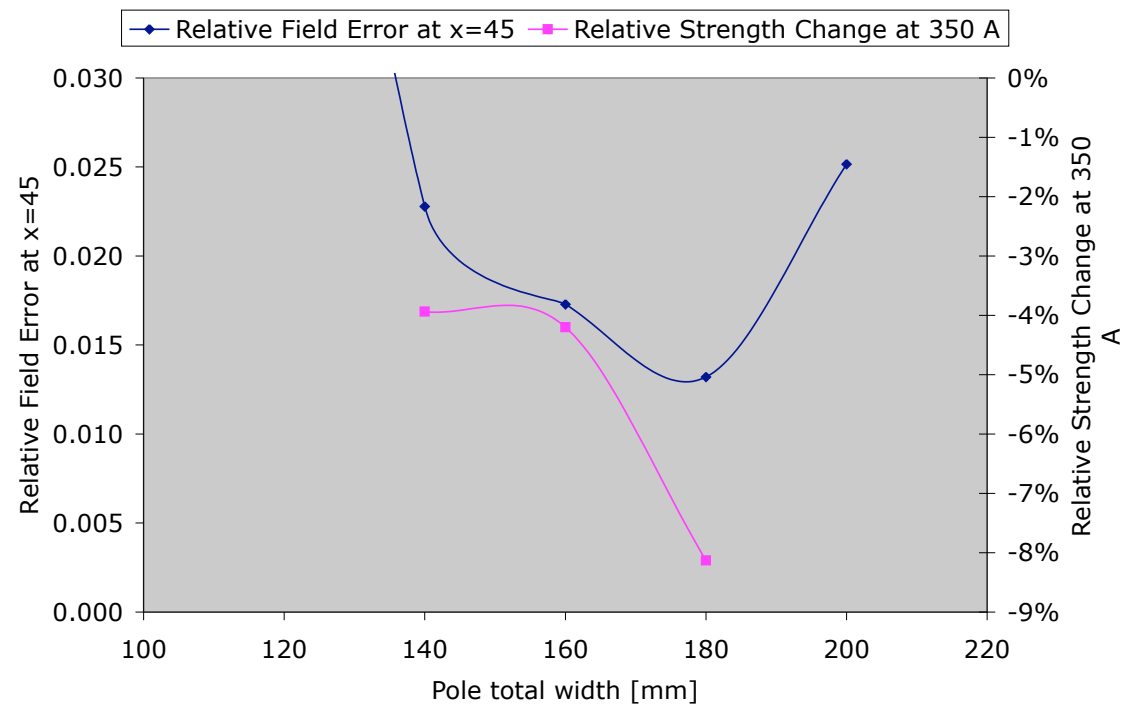

Figure 2: Calculated field quality and maximum strength as a function of pole width.

generally improved until the pole width was $200 \mathrm{~mm}$. The $180 \mathrm{~mm}$ pole width gives the best field quality and was adopted solely for that reason.

The model results also show that saturation becomes an important phenomena with wider poles where it effectively reduces the maximum strength the magnet can be run at. For the $180 \mathrm{~mm}$ pole width the saturation was found to be most pronounced in the pole root area, right next to the coil, and also main yoke as can be seen in Figure 3. The magnetic flux density is shown in this color contour plot of a one-quarter section of the model dipole. The maximum field is labeled MX. The pure yellow area of the yoke is between 1.89 and 2.2 T. This was calculated for $370 \mathrm{~A}$, substantially higher than the normal operating point. The predicted loss of maximum field can be accommodated using the trim coils and higher current trim power supplies than originally configured.

\subsection{Fabrication}

A somewhat unusual fabrication method was chosen for the pole modifications because the parallelism of the poles in magnetic gap was so critical to the getting a sufficiently uniform magnetic field. After rough machining the modified poles were assembled onto the yoke halves, and the halves were bolted together in the otherwise final configuration except the coils were absent. This assembly was put into a high precision EDM machine, and the entire profile was cut in one set-up. The tolerance stack-up, which would have ordinarily included size or form tolerances for: bottom pole, bottom pole to bottom yoke, bottom yoke 

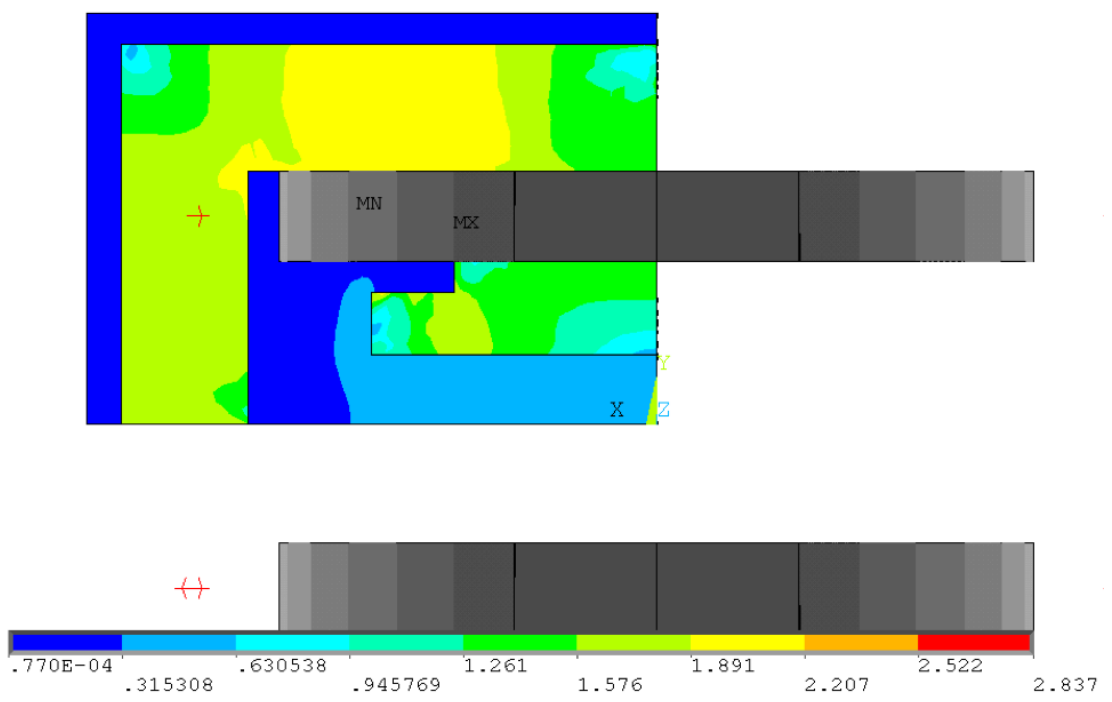

Figure 3: Magnetic flux density in the iron and gap.

Table 2: These measurements, (in inches), are taken from the mechanical inspection of the modified magnet gaps.

\begin{tabular}{lrr}
\hline \hline & BX12 & BX13 \\
\hline Parallelism & .0004 & .0003 \\
Flatness (top) & .00087 & .00092 \\
Flatness (bot) & .00076 & .00057 \\
\hline
\end{tabular}

to top yoke, top yoke to top pole and top pole size; was reduced to gap size and form only. Some mechanical measurements of the assembled magnets are reported in Table 2.

\subsection{Shimming}

Simply making the poles wider helps to reduce the field nonuniformity, but it is not enough to reach the target specification for field quality without further optimization. Fortunately we had some time in the schedule during which we could try to flatten the field profile using thin steel shims. The field profile of the modified dipoles was first measured without any shims in place and was found to be in reasonable agreement with the ANSYS prediction, but the sextupole and decapole multipoles were both negative and larger than desired. We then made up four shims out of steel strips 2 inches wide and a with a length to match the poles. They were arranged symmetrically in the dipole gap: top/bottom and 
right/left. The horizontal separation of the shims was optimized by iteratively adjusting their position, so that the measured integrated fields strengths were the same at the dipole center and a point about $34 \mathrm{~mm}$ from center. Based on a theoretical model of the effect of the shims, if the field at $34 \mathrm{~mm}$ was about the same as at the center, the shims were probably near their best positions. At first .010 inch thick shims were used, but they were found to be a bit too weak and were replaced by .015 inch thick shims. After a few iterations the optimal position was quickly found, and then a full field scan was performed.

During the search for the optimal position the shims were held in place by several screw/nut pairs that pushed against the top and bottom shim pairs. Once the optimal position was determined we sought to permanently attached the shims by spot welding them to the poles. However this was not successful, and two of the shims broke their spot welds and moved into the gap. Instead the shims were attached to the poles using epoxy. The epoxy bonds were tested by repeatedly rapidly ramping the magnet to $350 \mathrm{~A}$. No deformation was seen. Because the epoxy may eventually degrade, depending on the radiation levels, special shim blocks are being fabricated that will prevent the shims from falling into the gap should the epoxy ever fail. The shim blocks can be installed after the dipoles are in place. It is, however, unlikely that the epoxy will fail. The adjacent magnet coils are epoxy impregnated, will experience roughly the same radiation levels as the shims, and are not expected to fail. Coil damage due to radiation is only occasionally seen at accelerators, after a long time in particularly lossy areas, but that is not expected in the $\mathrm{BC} 1$ chicane.

\section{Measurements and Tests}

The principle measurements of interest were of field quality and of field strength as a function of main coil current. In addition, measurements were made of the strength of the trim current, the effect of disassembling and re-assembly the two dipole halves, and the thermal behavior of the dipoles at high currents. Some measurements of the effect of the vacuum chamber were made on the unmodified BX12 magnet and are reported here.

\subsection{Field Quality}

Ideally all electrons passing through a $\mathrm{BC} 1$ dipole should experience exactly the same magnetic field strength; or at least the difference in magnetic field strength should be linear in horizontal position, so that it can be corrected for by corrector quadrupoles. In practice field uniformity was determined, using a straight stretched wire, by measuring the integrated strength of the vertical magnetic field as a function of horizontal position. For each horizontal position the wire was repeatedly moved back and forth over range of $4 \mathrm{~mm}$, and the timeintegrated voltage induced by each move was recorded. The time-integrated voltage is proportional to the magnetic flux the wire cuts during the motion and is the integral of the vertical component of the magnetic field over its length, 
Table 3: Measured normalized multipole coefficients, evaluated at a reference radius of $20 \mathrm{~mm}$, for the modified BX12 and BX13 dipoles.

\begin{tabular}{ccc}
\hline \hline Coefficient & BX12 & BX13 \\
\hline$\left|b_{1} / b_{0}\right|$ & $1.51 \mathrm{E}-04$ & $-4.35 \mathrm{E}-05$ \\
$\left|b_{2} / b_{0}\right|$ & $-6.35 \mathrm{E}-04$ & $-6.86 \mathrm{E}-04$ \\
$\left|b_{3} / b_{0}\right|$ & $-1.40 \mathrm{E}-05$ & $-1.01 \mathrm{E}-05$ \\
$\left|b_{4} / b_{0}\right|$ & $6.42 \mathrm{E}-04$ & $5.62 \mathrm{E}-04$ \\
$\left|b_{5} / b_{0}\right|$ & $-2.60 \mathrm{E}-06$ & $7.76 \mathrm{E}-06$ \\
$\left|b_{6} / b_{0}\right|$ & $-1.15 \mathrm{E}-04$ & $-9.85 \mathrm{E}-05$ \\
\hline
\end{tabular}

multiplied by $4 \mathrm{~mm}$.

This method is well-suited for measuring these dipoles but is not perfect End effects are not seen since this method effectively averages the magnetic field over the entire length of the wire. Also, the radius of curvature of the electron path is about $2 \mathrm{~m}$ and is not exactly the straight line take by the wire.

Plots showing the results of such measurements are given in Figure 4, for BX12, and in Figure 5, for BX13. The field measured before shimming is also shown for comparison. Results for the two magnets are similar, but there tends to be more measurement noise in the BX12 measurements due to differences in the measurement apparatus. The final measurements for each magnet (after shims were epoxied into place) were fitted by a sixth order polynomial to yield the set of multipole coefficients given in Table 3. The effect of shimming is to make the sextupole term $\left(b_{2}\right)$ roughly equal and opposite of the decapole term $\left(b_{4}\right)$ in the region of interest. The odd numbered coefficients tend to be small because of the symmetry and reflect the overall geometrical accuracy of the top and bottom pole pieces.

The fitted curve for the integrated field deviation satisfies the target requirement of $\pm 5 \times 10^{-4}$ field variation over $\pm 26 \mathrm{~mm}$ that was described in Section 2.2. But a comparison of the measured multipoles with the PRD and ESD specifications, given in Table 1, shows that the modified dipoles do not quite satisfy the sextupole term requirement. However, the PRD/ESD specification was based on a calculation of the effect of each multipole independently, while the multipoles of the shimmed magnet act together to give a nearly flat field profile in the good field region.

\subsection{Field Strength}

Extending the pole width from $100 \mathrm{~mm}$ to $180 \mathrm{~mm}$ caused $80 \%$ more magnetic flux in the iron circuit and consequently saturation effects occur at a lower field strength. This was predicted by the $3 \mathrm{D}$ calculation based on the $\mathrm{BH}$ curve for 1008 steel. The actual steel in the magnets is combination of a very low carbon European grade steel and 1008 for the modified pole piece. A plot of the 

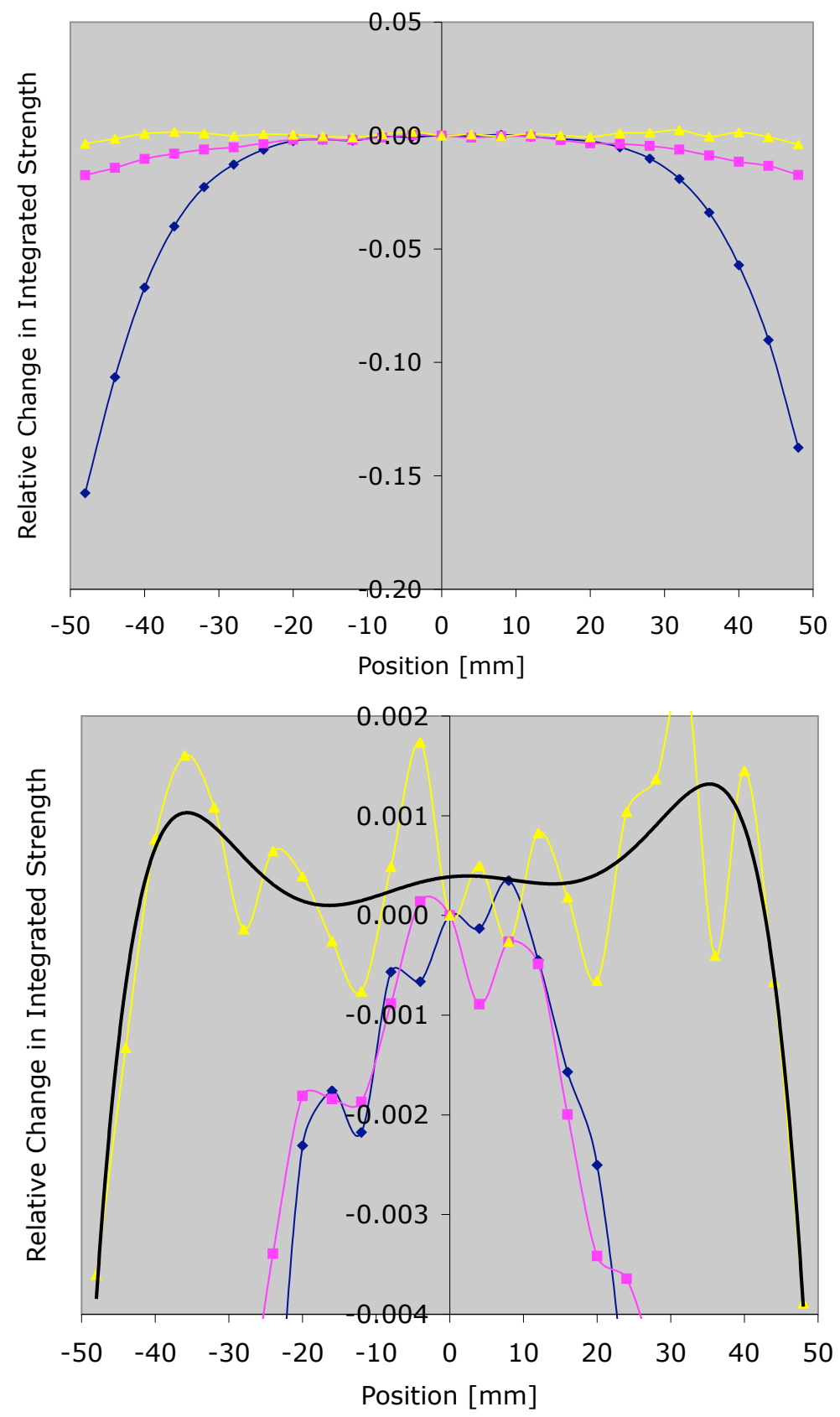

Figure 4: Field quality curves are plotted for BX12 before modification (blue), with $180 \mathrm{~mm}$ poles but before shimming (pink), and after shimming (yellow). A polynomial fit to the final configuration is also plotted (black). The upper plot shows the full range of data, the lower has the vertical scale expanded 33 times. 

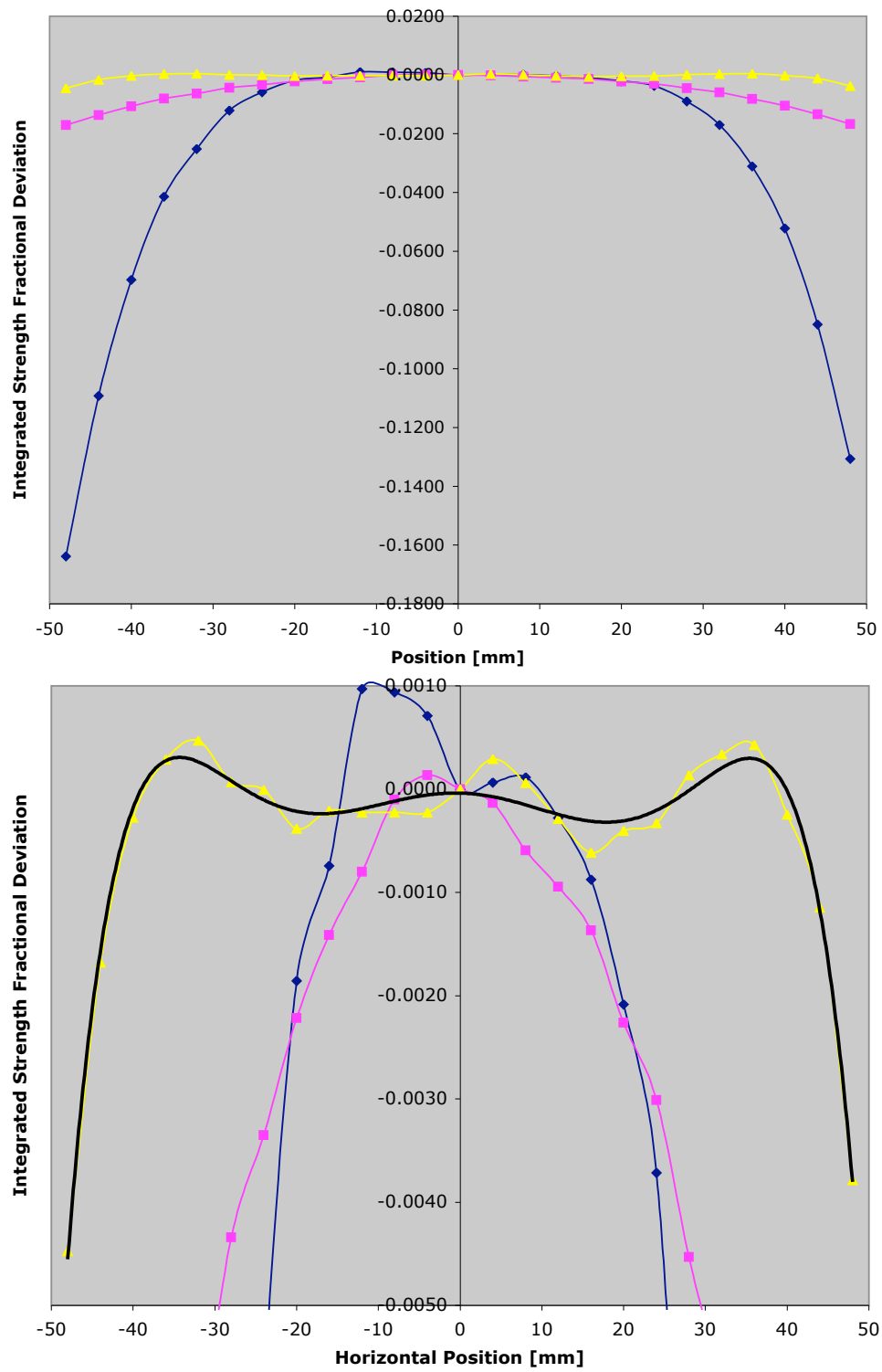

Figure 5: Field quality curves are plotted for BX13 before modification (blue), with $180 \mathrm{~mm}$ poles but before shimming (pink), and after shimming (yellow). A polynomial fit to the final configuration is also plotted (black). The upper plot shows the full range of data, the lower has the vertical scale expanded 33 times. 
measured integrated strength as a function of current is given in Figure 6 for the modified and un-modified BX12 magnet. Up to the normal operating point $\left(R_{56}=39 \mathrm{~mm}\right)$, the strength almost matches the original strength and is slightly higher for the same current, presumably due to the fact that the modified gap is $43 \mathrm{~mm}$ at the center of the pole while the original gap was $43.5 \mathrm{~mm}$. Around 240 A the effects of saturation start to be seen, and they are quite pronounced for currents near the power supply limit of $350 \mathrm{~A}$.

There are some performance implications due to the saturation. The specifications for the $\mathrm{BC} 1$ dipoles required that they be able to reach an integrated strength of $0.093 \mathrm{Tm}$, corresponding to the required maximum $R_{56}$ of $65 \mathrm{~mm}$ at $250 \mathrm{MeV}$ and an integrated strength of $0.112 \mathrm{Tm}$, to get to $R_{56}=65 \mathrm{~mm}$ at $300 \mathrm{MeV}$ operation. The modified magnets will only be able to reach the $250 \mathrm{MeV}$ requirement, and then only with large mismatch between the inner and outer dipoles. The coils were designed for $400 \mathrm{~A}$ maximum and tested up to 392 A. Because the current required to produce field strengths above about $0.1 \mathrm{Tm}$ increases rapidly with increasing field, it is very questionable if $0.112 \mathrm{Tm}$ can be reached without overheating the coils.

Trim windings on each of the $\mathrm{BC} 1$ dipoles are used to precisely match the strength of the four magnets. Since the two inner dipoles were substantially modified it was especially important to check if the trim windings and the associated power supplies were still capable of making the match. Measurements of the integrated strength as a function of effective main coil current around the operating point for BX13 are shown in Figure 7. Virtually the same performance was observed for BX12, although the offset at zero main current was slightly different. The effective main coil current is the current that would be needed in the main coil to get the observed integrated strength, assuming there is no current in the trim coil. To the first approximation the ampere-turns of the trim coil can be equated with ampere-turns in the main coil. However, the data show that a better fit is obtained if 45 turns are assumed for the trim coil instead of the 46 turns that are called out in the drawings. Also of note is that there is potentially a $1 \%$ percent error in integrated strength that can result if the magnet is on the wrong hysteresis curve. Examination of the hysteresis curves reveals that it takes roughly $5 \mathrm{~A}$ change in current in the opposite direction to go from one branch of the curve to the other.

\subsection{Vacuum Chamber Effect}

All of the measurements so far discussed were done without the presence of the vacuum chamber. Measurements on the unmodified BX12 dipole, done in September 2006, indicate the presence of the vacuum chamber tends to raise the field in the gap about $0.5 \%$ and possibly causes some field distortion as well. This is illustrated in Figure 8. Though the data are noisy, there appears to be an induced distortion which, if real, could be large enough to degrade the field quality of the shimmed modified dipole. 

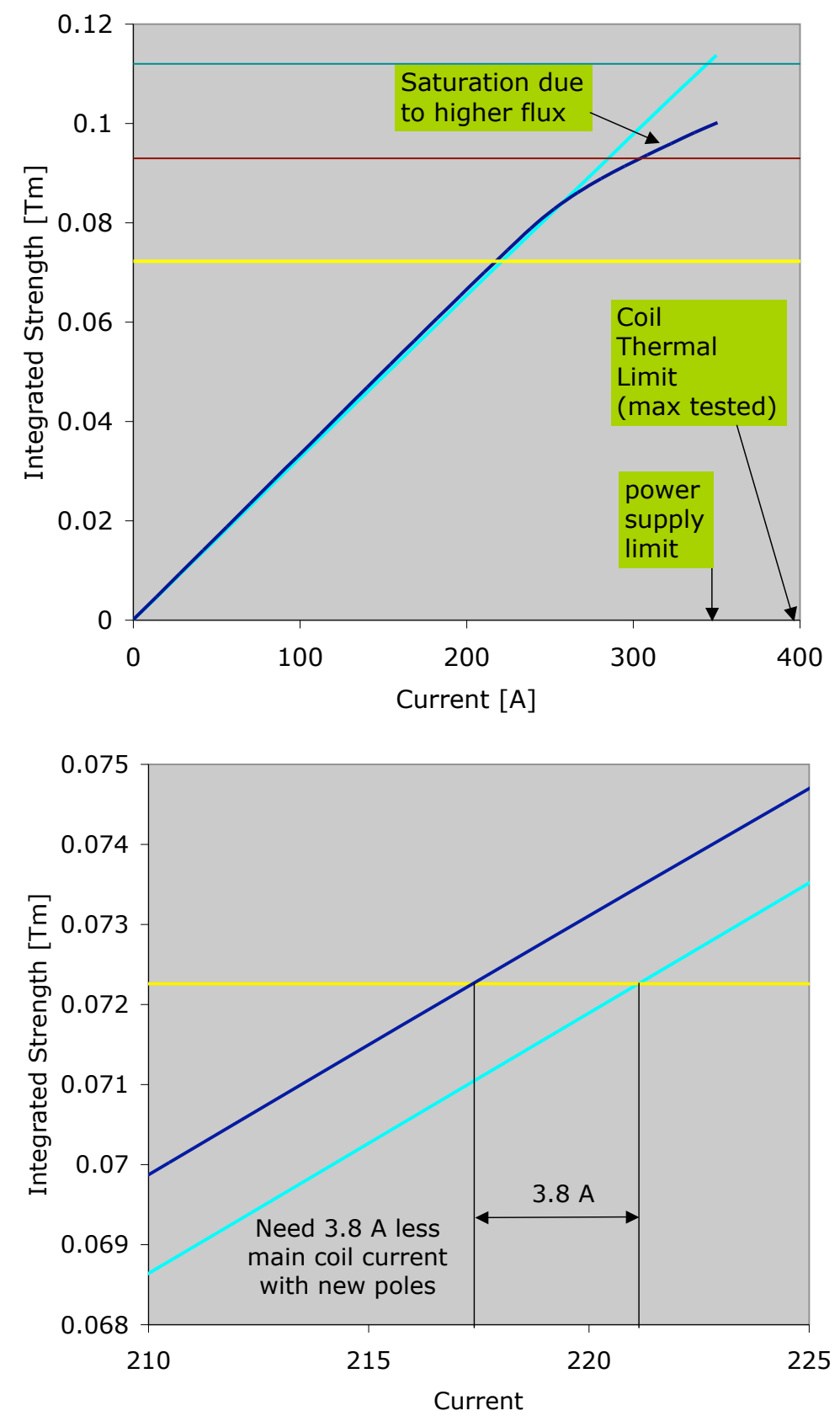

Figure 6: Integrated strength as a function of main coil current for the unmodified (light blue) and modified (dark blue) BX12 magnet. The normal operating point is shown by the yellow horizontal line, the red line corresponds to $R_{56}=65 \mathrm{~mm}$ at $250 \mathrm{MeV}$ and the blue-green line is for $R_{56}=65 \mathrm{~mm}$ at $300 \mathrm{MeV}$. A close-up of the normal operating point is shown in the lower plot. 


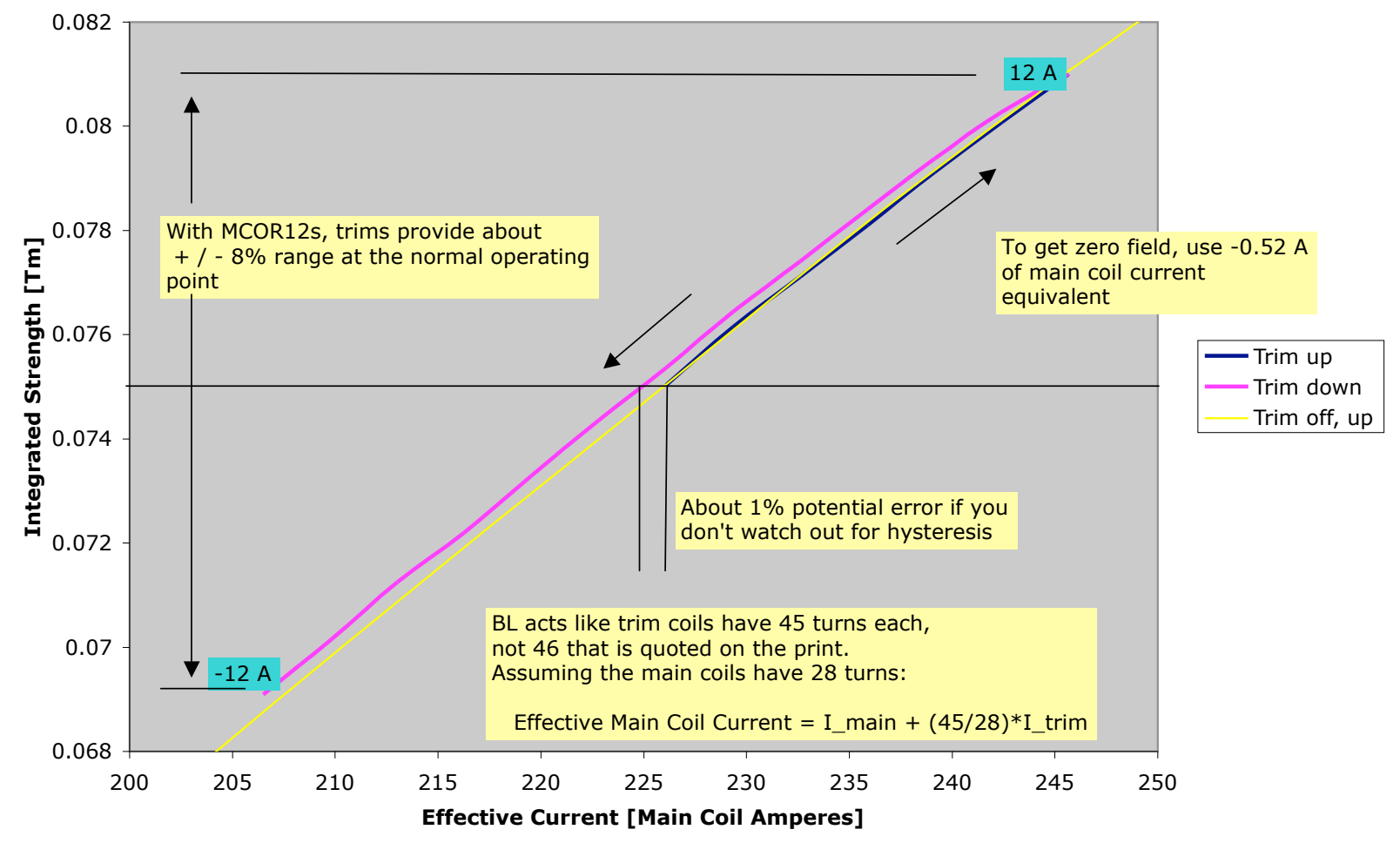

Figure 7: Integrated strength as a function of Effective Main Coil current for BX13. 


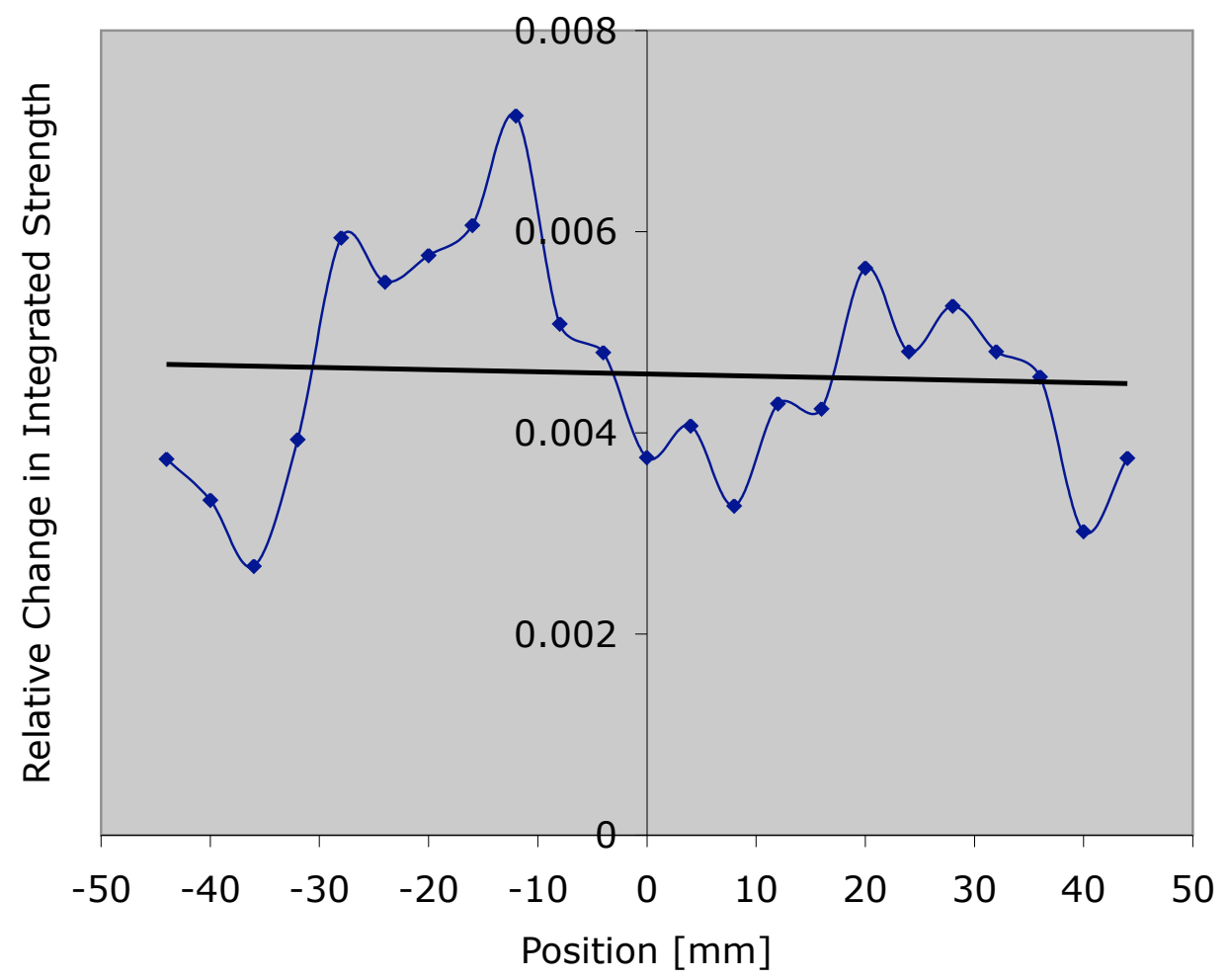

Figure 8: Change in relative integrated strength as a function horizontal position after inserting a substitute vacuum chamber in the gap. A linear fit to the data is given for reference. Data is for the unmodified BX12 dipole from September 2006 . 


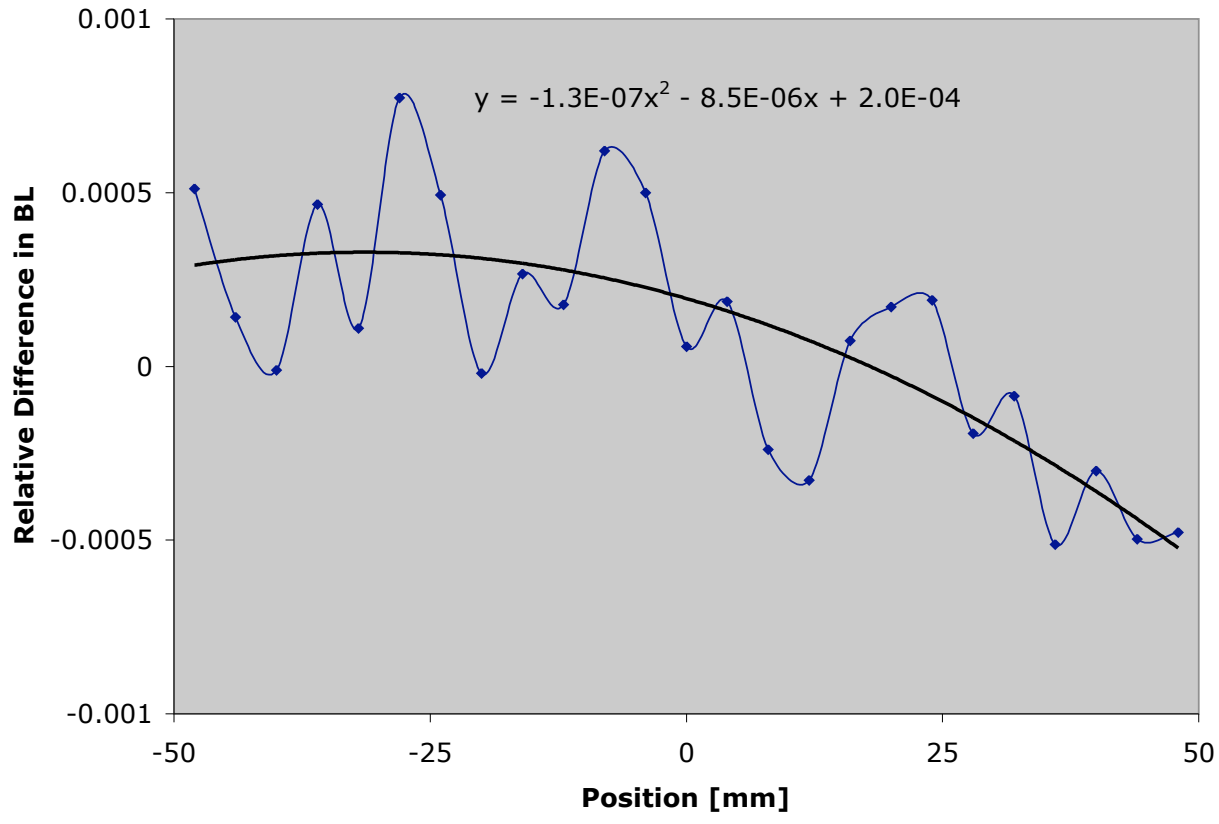

Figure 9: After the two yoke halves were reassembled, the relative difference in the integrated strength (After - Before) was measured and is plotted as a function of horizontal position. Error bars are not shown for clarity.

\subsection{Assembly Test}

Before and After measurements of integrated strength as a function of position were made to test how well the field is reproduced after a dipole is disassembled and re-assembled. The results, shown for BX13 in Figure 9, indicate a linear change of $8.5 \times 10^{-6}$ in relative field strength per millimeter of horizontal positon is introduced by the operation. This corresponds to a geometrical change in the angle between the poles of $43 \times 8.5 \times 10^{-6}=3.7 \times 10^{-4}$ and a quadrupole coefficient change of $\left|\Delta b_{1} / b_{0}\right|=20.5 \times 10^{-6}=1.7 \times 10^{-4}$. The quadrupole coefficient change is about the same as the total ESD tolerance for the quadrupole coefficient, but much less than the PRD tolerance. The inferred wedge angle is equivalent to a gap change of almost .003 inches across the pole width, so the diassembly/re-assembly operation may not be mechanically well-reproducible but seems to have been adequate in this one test.

\subsection{Thermal Tests}

Stringent thermal tests were required for the modified magnets because of the potentially higher current operation resulting from increased saturation. The 
BX12 dipole was run up to $392 \mathrm{~A}$ on the main coil (almost the engineering design limit) and simultaneously $12 \mathrm{~A}$ on the trim coil for one hour. The overall temperature rise in cooling water was less than $5 \mathrm{deg} \mathrm{C}$, and the temperature rise of a point measured on the coil was even less.

\section{Beam Calculations}

We simulated the effect on beam emittance of the remaining field non-uniformities. For simplicity the simulations used the measured (unmodified) BX12 field for the (also unmodified) BX11 dipole and the measured field of the (modified) BX13 dipole for the (modified) BX14 dipole. The other parameters were adopted according to the LINAC MAD deck.

The horizontal variation of the field in the simulation was the matched to the variation in the integrated measured field. However, the $z$-dependence was modeled assuming a hard edge approximation. The electrons see the effect of the transverse field variation through the electron motion. Inside the dipole, as the electron advanced in $z$, its $x$-coordinate also changed.

As illustrated in the previous sections, the horizontal $x$-dimension of the electron beam in the inner two dipoles was quite wide, and so the beam sampled their high-order nonlinear field components. The horizontal $x$-dimension, $\sigma_{x}$, was mostly determined by the electron correlated energy spread, $\sigma_{\delta}$, through the relation $\sigma_{x} \sim \eta_{x} \sigma_{\delta}$, where $\eta_{x}$ is the horizontal dispersion function. After the harmonic cavity linearizes the longitudinal phase space, the correlated energy spread is $\sigma_{\delta} \sim h \sigma_{z}$, where $h$ is the relative energy deviation slope along the electron bunch. Hence the electron bunch transverse $x$-dimension scales as $\sigma_{x} \sim$ $\eta_{x} h \sigma_{z}$. So a longer electron bunch and a larger relative energy deviation slope lead to a wider transverse $x$-dimension in the chicane and therefore lead to a larger emittance growth. In Figure 10 we show the emittance growth as a function of $h$, where the nominal bunch length $\sigma_{z}=830 \mu \mathrm{m}$ is used. For our nominal compression, we need $h \sim 19 \mathrm{~m}^{-1}$. According to Figure 10 we find that we should expect about $\Delta \epsilon_{n} / \epsilon_{n} \sim 8 \%$ emittance growth with the modified dipole magnets. 


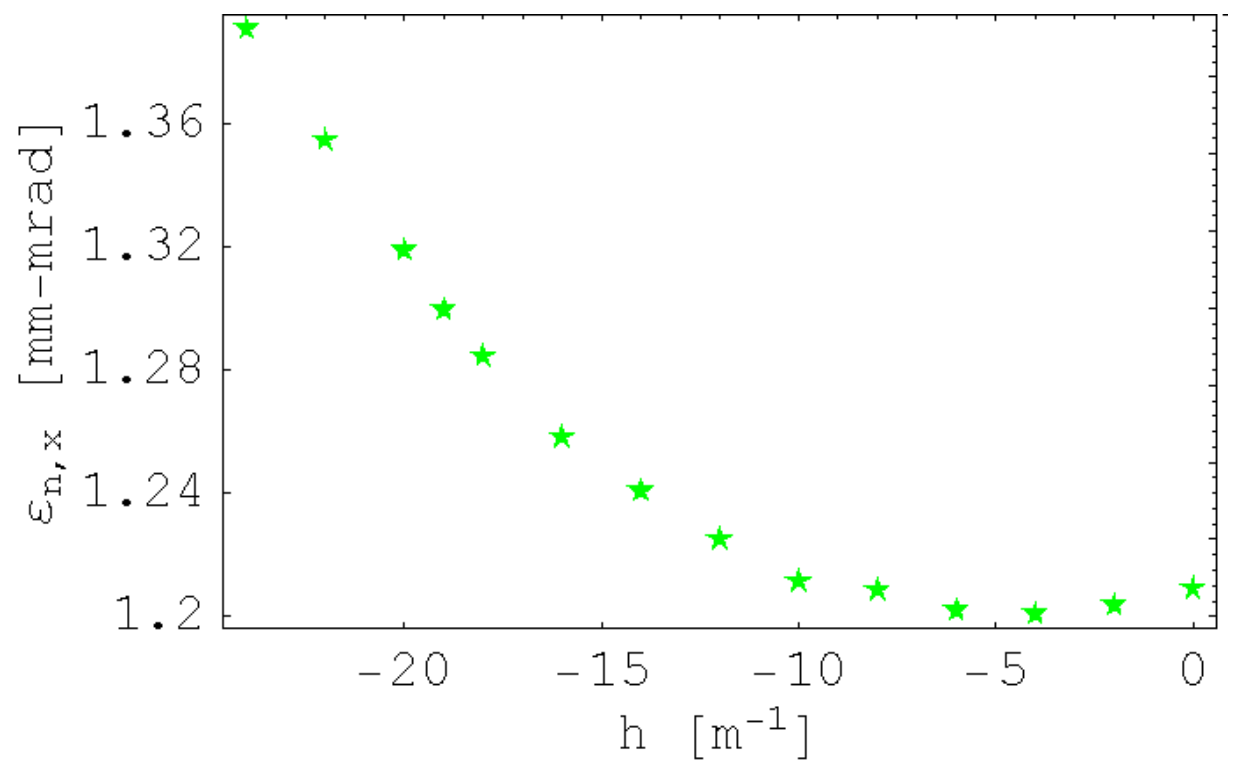

Figure 10: Based on the modified dipole field measurements, the calculated normalized emittance after $\mathrm{BC} 1$ is plotted as a function of the compression parameter. 